\title{
Caracterização dos Argissolos amarelos com adição de resíduos de madeira: uma alternativa de uso como cobertura em solos da Amazônia paraense ${ }^{1}$

\author{
Characterization of yellow Argisoils with added wood residues: \\ an alternative use as soil cover in the state of Pará
}

\author{
Kátia Fernanda Garcez Monteiro \\ Dirse Clara Kern II \\ Tarcísio Ewerton Rodrigues III \\ Maria de Lourdes Pinheiro Ruivo "I \\ Paulo Roberto Silva Farias ${ }^{\mathrm{N}}$
}

\begin{abstract}
Resumo: Em solos altamente intemperizados, como ocorre nos trópicos, a razão de seu empobrecimento está relacionada à lixiviação dos nutrientes das camadas superficiais. Uma alternativa para minimizar esta perda de nutrientes seria a utilização de coberturas orgânicas verdes ou mortas (mulch) nestes solos. Nesse sentido, com o intuito de caracterizar os aspectos morfológicos, físicos e químicos dos solos com e sem adição de resíduos de madeira como cobertura, sob as culturas de paricá (Schizolobium amazonicum) e pupunha (Bactris gasipaes), foram selecionados e coletados 32 amostras de 4 perfis de solo das áreas de plantio de paricá e pupunha com e sem adição de resíduos de madeira. A utilização de resíduos de madeira como cobertura favoreceu a diminuição da densidade, com valores entre 1,19 a 1,33 kg. $\mathrm{dm}^{-3}$ para os solos com adição de resíduos, e de 1,19 a 1,67 kg. $\mathrm{dm}^{-3}$ para os solos sem adição. A mesma influência pode ser percebida no aumento da porosidade nos solos com adição de material, com valores de 0,48 a $0,46 \mathrm{~m}^{3} \cdot \mathrm{m}^{-3}$ nos horizontes superficiais, enquanto que os solos sem resíduos demonstraram resultados de 0,35 a $0,38 \mathrm{~m}^{3} \cdot \mathrm{m}^{-3}$. Os resultados de $\mathrm{pH}$ em água, nos solos com cobertura de resíduos de madeira, variaram de 4,6 a 6 e, nos solos sem adição, de 4,6 a 5,2, podendo ser verificado que a adição de resíduos de madeira favoreceu o aumento do $\mathrm{pH}$ no solo. Os valores mais elevados para a saturação de bases foram observados também para os solos com utilização de resíduos de madeira como cobertura, cujos valores variaram entre $26 \%$ a 54\%, enquanto que os perfis sem adição de material orgânico apresentaram variação entre $26 \%$ a 39\%. O uso adequado de resíduos de madeira no solo desempenhou papel importante na disponibilidade de alguns nutrientes, como o cálcio e o magnésio, e diminuiu a acidez dos solos. Assim, a utilização de resíduos de madeira como cobertura no solo em sistemas agroflorestais é satisfatória por permitir uma destinação adequada para estes materiais, além de melhorar as condições ambientais dos solos amazônicos.
\end{abstract}

Palavras-chave: Resíduos de madeira. Matéria orgânica. Propriedades físicas e químicas de solos.

\footnotetext{
I. Museu Paraense Emíio Goeldi. Belém, Pará, Brasil (kfgarcez@amazon.com.br).

II Museu Paraense Emílio Goeldi. Pesquisadora. Belém, Pará, Brasil (kern@museu-goeldi.br) (ruivo@museu-goeldi.br).

III Empresa Brasileira de Pesquisa Agropecuária. Pesquisador. Belém, Pará, Brasil (rodrigues@cpatu.embrapa.br).

Iv Universidade Federal Rural da Amazônia. Instituto de Ciências Agrárias. Professor. Belém, Pará, Brasil (paulo.farias@ufra.edu.br).

1 Parte da dissertação de mestrado do primeiro autor, apresentada à coordenação do curso de mestrado em Agronomia da Universidade Federal Rural da Amazônia.
}

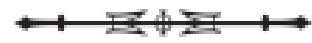


Caracterização dos Argissolos amarelos com adição de resíduos de madeira...

Abstract: In soils highly intemperic happens in the tropics, the reason of impoverishment is related to the lixiviation of the nutrients of the superficial layers. An alternative to minimize this loss of nutrients would be the use of organic coverings in these soils, be covering green or dead (mulch). In that sense, with the intention of characterizing the morphologic aspects, physical and chemical of the soils with and without addition of wood residues as covering, under the paricá (Schizolobium amazonicum) and pupunha culture (Bactris gasipaes). Were selected and collected 32 samples of 4 profiles of soil of the areas of paricá and pupunha planting with and without addition of wood residues for evaluation of their morphologic characteristics, physics and chemistries. The use of wood residues as covering favored for decrease of the density with values among 1,19 to 1,33 kg. $\mathrm{dm}^{-3}$ for the soils with addition of residues, while the soils without addition of wood residues the values were from 1,19 to 1,67 kg. $\mathrm{dm}^{-3}$. The same influence can be noticed for the increase of the porosity in the soils with material addition with values from 0,48 to $0,46 \mathrm{~m}^{3} \cdot \mathrm{m}^{-3}$ in the superficial horizons, while the soils without residues, these demonstrated results from 0,35 to $0,38 \mathrm{~m}^{3} \cdot \mathrm{m}^{-3}$. The $\mathrm{pH}$ values in water, for the soils with covering of wood residues varied from 4,6 to 6,0 . To the soils without addition of wood residues, these presented values from 4,6 to 5,2. Could be verified that the addition of wood residues favored in the increase of the $\mathrm{pH}$ in the soil. The highest values for the saturation of bases were also observed for the soils with use of wood residues as covering, the values varied inside of these profiles, among 26 to $54 \%$. While that for the profiles without addition of organic material presented variation among 26 to 39\%. The appropriate use of wood residues in the soil played important part in the readiness of some nutritious as the calcium and the magnesium, reduced the acidity of the soils. Like this, the use of wood residues as covering in the soil in agroforests systems is satisfactory for allowing an appropriate destination for these materials besides improving the environmental conditions of the Amazon soils.

Keywords: Wood residues. Organic matter. Soil physical and chemical properties.

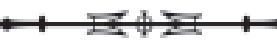




\section{INTRODUÇÃO}

Na Amazônia, onde grande parte das terras ocupadas estão destinadas à agricultura ou à pecuária, a erosão resultante da alta pluviosidade e o tipo de preparo do solo com as queimas podem eliminar rapidamente a camada superficial do solo considerada mais fértil após a remoção da cobertura vegetal.

Nesse contexto, a cobertura vegetal desempenha um papel importante na disponibilidade de nutrientes, pois a maior parte da capacidade de troca de cátions (CTC) destes solos é devido aos colóides orgânicos. A utilização de material orgânico como cobertura no solo pode favorecer alguns elementos essenciais (cálcio, magnésio, potássio, fósforo etc.) para o bom desenvolvimento da planta (GREENLAND, 1981).

O aproveitamento do solo natural com adição de resíduos vegetais pode conduzir a uma elevação do conteúdo de matéria orgânica em solos de regiões tropicais, onde o aporte do material orgânico é significativamente maior do que em regiões de clima temperado (RAIJ, 1991). Certamente, além dos aspectos químicos, há de se considerar, também, os aspectos econômicos, sociais e ambientais envolvidos neste processo, visto que os mesmos são de vital importância para a harmonia e sustentação do sistema agrícola como um todo. Dessa forma, o uso de materiais orgânicos no solo deixa de constituir um aspecto meramente pontual e adquire conotações sistêmicas, proporcionando a melhoria dos atributos edáficos pela racionalização das estratégias de manejo do sistema solo-planta ao longo do tempo (MUZILLI, 1996).

Pesquisas relacionadas ao comportamento da matéria orgânica sobre as propriedades físicas do solo têm demonstrado que, ao favorecer a maior agregação de partículas, a matéria orgânica contribui para a melhoria da porosidade, beneficiando a aeração, a infiltração e o armazenamento da água no solo (SANCHEZ, 1976; VIEIRA et al., 2000), assim como poderá nortear práticas de manejo e uso racional dos mesmos. Outro fator a ser considerado são os aspectos físicos do solo, que poderão ser avaliados através da mensuração de algumas características físicas, como porosidade, densidade, resistência e teor de umidade (FULLER; WARRICK, 1986). Esses aspectos físicos mudam com as práticas e sistemas de cultivo, a partir da remoção da vegetação natural, alterando, dessa maneira, as relações solo-planta (BAENA; DUTRA, 1979,1981; FELLER; BEARE, 1997).

Um exemplo da problemática apresentada é o caso do município de Tailândia, no estado do Pará, considerado um dos maiores pólos madeireiros do estado e que já perdeu mais de 35\% da sua cobertura vegetal original. Segundo dados da imprensa local, grande parte da matéria-prima das serrarias é desperdiçada e cerca de $50 \%$ da madeira cortada não é aproveitada pelas empresas, que acabam adotando o processo de incineração ou acumulando em locais inadequados, causando assoreamento da rede fluvial e diminuição de áreas para a agricultura.

A concentração de serrarias tem chamado a atenção, tanto da população como dos órgãos públicos, devido à grande produção de rejeitos que estas atividades acumulam e às discussões de como eliminar ou reaproveitar este material. Os proprietários têm adotado como uma das alternativas, a queima a céu aberto, causando problemas à saúde das comunidades sobre a área de influência deste tipo de atividade. $\bigcirc$ processo de incineração dos rejeitos de serrarias também lança grande quantidade de $\mathrm{CO}_{2}$ na atmosfera, contribuindo para o aumento do fenômeno de ilhas de calor e o efeito estufa, o que afeta diretamente a população e o ambiente urbano com o desconforto ambiental.

A utilização de resíduos de madeira, provenientes de serrarias, como cobertura no solo, poderá apontar novas alternativas de usos de materiais orgânicos nos solos amazônicos e estabelecer relações mais harmoniosas entre o setor madeireiro

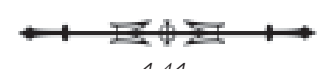


e o meio ambiente por meio da adoção de práticas de mecanismo de desenvolvimento limpo (MDL), ao incorporar os resíduos da atividade madeireira em seus sistemas de reflorestamentos, agregando, portanto, valor aos materiais que seriam incinerados, causando sérios problemas de ordem ambiental.

Este trabalho teve como objetivo estudar o efeito da adição de resíduos de madeira nas principais propriedades físicas e químicas dos solos, quando utilizados como cobertura em áreas de reflorestamento.

\section{MATERIAL E MÉTODOS}

\section{Caracterização da área de estudo}

O estudo foi realizado na área experimental da empresa de laminação Tailâminas Plac Ltda, localizada no município de Tailândia, mesorregião nordeste do estado do Pará (Figura 1).

As características ambientais da área estão representadas por um clima do tipo Ami da classificação de Köppen, com normal pluviométrica de $2.590 \mathrm{~mm}$, e temperatura média anual de $28^{\circ} \mathrm{C}$. Nas áreas não cultivadas predomina a vegetação do tipo floresta equatorial subperenifólia densa; o relevo predominante na área é o plano e suave ondulado; e os principais solos encontrados na área são os argissolos amarelos distróficos de texturas média e argilosa, de baixa fertilidade química, porém, com boas propriedades físicas, bem drenados, friáveis e profundos (RODRIGUES, 2001).

Para a classificação e caracterização das propriedades físicas dos solos foram abertos 4 perfis de solos nas culturas de paricá (Schizolobium amazonicum) e de pupunha (Bactris gasipaes), com 2 perfis amostrados com adição de resíduos de madeira e 2 perfis em locais de solo sem adição de resíduos de madeira. Os solos foram coletados e descritos conforme metodologia proposta por Lemos e Santos (1999).

A adição foi realizada no ano de 2000, quando o proprietário da empresa Tailâminas selecionou 1 ha de uma área total plantada de 18 ha de paricá (Schizolobium amazonicum) para adição de resíduos de madeira, adicionando à superfície do solo aproximadamente $60 \mathrm{~m}^{3}$ de resíduo de madeira

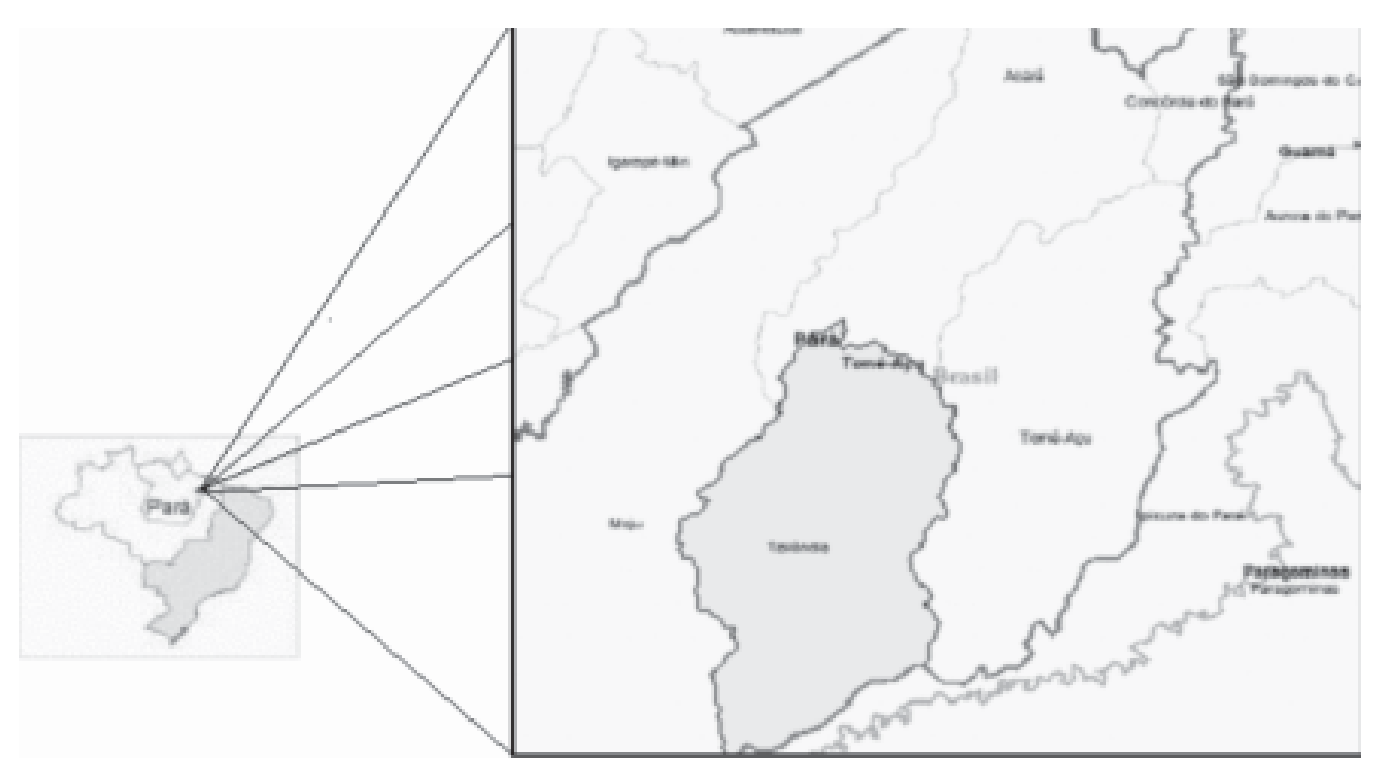

Figura 1. Mapa de localização do município de Tailândia. Fonte: IBGE (2000).

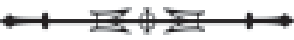

142 
(lâmina triturada e pó de serragem) e 1 ha em solos naturais, sem qualquer tipo de adubação.

Amostras indeformadas de solo foram coletadas em perfis abertos nessas áreas, no centro dos horizontes, com uma profundidade variando de 0 a $190 \mathrm{~cm}$, com a utilização de anéis volumétricos de 100 cc de capacidade, totalizando 32 amostras. No mesmo local também foram coletadas amostras deformadas para a análise granulométrica e a densidade de partículas.

As amostras foram secas ao ar, destorroadas e passadas em peneira de $2 \mathrm{~mm}$ de abertura de malha, obtendo-se a terra fina seca ao ar (TFSA), a partir da qual foram realizadas as análises laboratoriais. As análises físicas constaram de granulometria, densidade do solo, densidade de partícula e porosidade total e as análises químicas constaram de $\mathrm{pH}$ em $\mathrm{H}_{2} \mathrm{O}$ e KCl$, \mathrm{Ca}^{2+}, \mathrm{Mg}^{2+}, \mathrm{K}, \mathrm{P}, \mathrm{Na}, \mathrm{Al}^{3+} \mathrm{C}$, $\mathrm{MO}, \mathrm{N}$. Os métodos utilizados são baseados no Manual de Métodos de Análises de Solos (EMBRAPA, 1999). A granulometria do solo foi determinada pelo método da pipeta e a dispersão da amostra ( $10 \mathrm{~g}$ de TFSA) foi realizada com hidróxido de sódio 0,1N.

A densidade do solo (Ds) foi determinada pelo método do anel volumétrico (cilindro de Uhland); a densidade de partícula (Dp) foi determinada pelo método do balão volumétrico e a porosidade total (Pt) foi calculada pela fórmula: pt=(1-Ds/Dp).100.

$\mathrm{Na}$ caracterização química, determinaram-se os pHs em água e em solução de $\mathrm{KCl} 1 \mathrm{~N}$ em suspensão solo/solução na proporção 1:2,5; extraíram-se cátions trocáveis por $\mathrm{KCl} 1 \mathrm{~N}$ e foram quantificados por espectrometria de absorção atômica $\left(\mathrm{Ca}^{2+} \mathrm{e}\right.$ $\mathrm{Mg}^{2+}$ ) e fotometria de chama $\left(\mathrm{Na}^{+}\right.$e K$\left.^{+}\right) ; \mathrm{Al}^{3+}$ foi extraído por $\mathrm{KCl} 1 \mathrm{~N}$ e determinado por titulometria com $\mathrm{NaOH} ; \mathrm{H}+\mathrm{Al}^{3+}$ foram extraídos por acetato de cálcio $0,5 \mathrm{~N}$ a pH 7,0 e quantificados por titulometria com $\mathrm{NaOH}$. O P disponível foi extraído seguindo o método Mehlich $\left(\mathrm{HCl} \mathrm{0,05} \mathrm{N}+\mathrm{H}_{2} \mathrm{SO}_{4}\right.$ $0,0125 \mathrm{~N}$ ) e quantificado por colorimetria e $\mathrm{O}$ carbono orgânico determinado pelo método Walkley-Black.

\section{RESULTADOS E DISCUSSÃO}

\section{Características morfológicas e físicas}

As características morfológicas e físicas dos solos com e sem adição de resíduos de madeira são apresentados na Tabela 1.

Em geral, apresentam horizontes B textural, são bem drenados, profundos e com seqüência de horizontes do tipo $A_{p}, A B, B A$. Apresentam coloração variando de bruno escuro a amarelo brunado nos matizes 7,5 YR a 10 YR; a classe de textura varia de areia franca a franco-argilo-arenosa no horizonte Ap e franco-argilo-arenosa a argilosa no horizonte $\mathrm{B}_{\mathrm{t}}$; a estrutura é fraca, pequena e média granular no horizonte Ap e em forma de blocos subangulares no horizonte $B$; e a consistência do solo é muito friável a friável quando úmida, variando de ligeiramente plástica a plástica e ligeiramente pegajosa a pegajosa quando o solo está molhado.

A relação entre silte e argila com valores inferiores a 0,6 (Tabela 2) demonstra o elevado estado de intemperismo destes solos. Isto já era esperado por se tratar de Argissolos, desenvolvidos de material de origem retrabalhados, com a virtual ausência de minerais primários pouco resistentes aos processos intempéricos (RODRIGUES, 2001).

O conteúdo da fração argila dispersa em água variou de 20 a $280 \mathrm{~g} \mathrm{~kg}^{-1}$ de solo, mostrando a tendência de aumentar com a profundidade (Tabela 2). Observou-se, ainda, uma concentração desta fração nos horizontes $B A$, nos perfis P1 e P2, e nos horizontes, $\mathrm{AB}$ e $\mathrm{BA}$ nos perfis $\mathrm{P} 2$ e $\mathrm{P} 3$.

Ocorre também um incremento do conteúdo da fração argila do horizonte Ap para o horizonte BA, evidenciando a existência de relação textural $B / A$, característica para definir a presença de B textural nesses solos, conforme Embrapa (1999). O manejo das áreas com a cultura do paricá (P1 e P2) utilizando gradagem entre as fileiras de plantas concorreu para um maior acúmulo da fração argila no horizonte

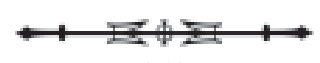


Caracterização dos Argissolos amarelos com adição de resíduos de madeira...

Tabela 1. Características morfológicas e físicas dos solos sob as culturas de pupunha e paricá.

\begin{tabular}{|c|c|c|c|c|c|c|}
\hline Horiz. & Prof. $(\mathrm{cm})$ & Cor & Textura & Consistência & Estrutura & Classe textural \\
\hline \multicolumn{7}{|c|}{ PERFIL 1 - PARICÁ COM RESÍDUO: ARGISSOLO AMARELO DISTRÓFICO TÍPICO } \\
\hline $\begin{array}{l}A p \\
A B\end{array}$ & $\begin{array}{l}0-13 / 15 \\
13 / 15-25\end{array}$ & $\begin{array}{l}10 Y R 5 / 4 \\
10 Y R 5 / 6\end{array}$ & $\begin{array}{l}\text { Fran. Aren. } \\
\text { Fran. Arg. Aren. }\end{array}$ & $\begin{array}{l}\text { lig. Peg. e lig. Plas. } \\
\text { lig. Peg. e lig. Plas. }\end{array}$ & $\begin{array}{l}\text { G. Fra,. Peq. Méd. } \\
\text { F. P. M. Bs }\end{array}$ & $\begin{array}{l}\text { Fran. Aren. } \\
\text { Fran. Arg. Aren. }\end{array}$ \\
\hline BA & $25-45$ & $\begin{array}{l}10 \text { YR 6/8 } \\
\text { FIL } 2 \text { - PARIC }\end{array}$ & $\begin{array}{l}\text { Franco Arg.Aren. } \\
\text { SEM RESÍDUO: ARGI }\end{array}$ & $\begin{array}{l}\text { lig. Peg. e lig. Plas. } \\
\text { ISSOLO AMARELO DISTR }\end{array}$ & $\begin{array}{l}\text { F.P.M. } \\
\text { RÓFICO TÍPICO }\end{array}$ & Franco Aren. \\
\hline $\begin{array}{l}A p \\
A B\end{array}$ & $\begin{array}{l}0-10 \\
10-25\end{array}$ & $\begin{array}{l}10 Y R 4 / 3 \\
10 Y R 4 / 4 \\
10 Y R 5 / 4\end{array}$ & $\begin{array}{l}\text { Areia Franca } \\
\text { Franco argilo arenosa } \\
\text { Franco argilo arenosa }\end{array}$ & $\begin{array}{l}\text { Friável Não pla. Não peg. } \\
\text { Firme } \\
\text { Lig. Firme, plas e peg }\end{array}$ & $\begin{array}{l}\text { G. Fra. Peq. Méd. } \\
\text { Lig. Pl. e Lig. Peg. } \\
\text { Gran. Maciço } \\
\text { F. P. M. Bs }\end{array}$ & $\begin{array}{l}\text { Areia Fran. } \\
\text { F. P. M. } \\
\text { Fran. Aren. } \\
\text { Fran. Arg. Aren. }\end{array}$ \\
\hline \multicolumn{7}{|c|}{ PERFIL 3 - PUPUNHA COM RESÍDUO: ARGISSOLO AMARELO EUTRÓFICO TÍPICO } \\
\hline Ap & $0-7$ & 10YR $3 / 2,5$ & Franco arenosa & Lig. Firme & $\begin{array}{l}\text { F. P. M. } \\
\text { Granular }\end{array}$ & $\begin{array}{l}\text { Franco Argilo } \\
\text { Arenosa }\end{array}$ \\
\hline$A B$ & $7-20$ & 10YR 5/6 & Franco arenosa & Lig. Firme & $\begin{array}{l}\text { F. P. M. } \\
\text { Subangular }\end{array}$ & $\begin{array}{l}\text { Franco Argilo } \\
\text { Arenosa }\end{array}$ \\
\hline BA & $20-31$ & 10YR 5,5/6 & Argila arenosa & Friável & F. P. M. Subangular & Argilosa \\
\hline \multicolumn{7}{|c|}{ PERFIL 4 - PUPUNHA SEM RESÍDUO: ARGISSOLO AMARELO DISTRÓFICO TÍPICO } \\
\hline Ap & $0-10$ & $\begin{array}{l}10 Y R 4 / 3 \\
10 Y R 3 / 3\end{array}$ & Franco arenoso & Firme & $\begin{array}{l}\text { Lig. Peg. Lig Plas. } \\
\text { Fraca a mod., } \\
\text { P. Med. Granular }\end{array}$ & $\begin{array}{l}\text { Franco Argilo } \\
\text { Arenoso }\end{array}$ \\
\hline$A B$ & $10-26$ & $\begin{array}{l}10 Y R 5 / 6 \\
10 Y R 4 / 6\end{array}$ & Franco argilo arenosa & Firme & $\begin{array}{l}\text { Lig. Peg. Lig Plas } \\
\text { Bs }\end{array}$ & $\begin{array}{l}\text { F. P. M. } \\
\text { Franco Argilo } \\
\text { Arenoso }\end{array}$ \\
\hline BA & $26-48$ & $\begin{array}{l}10 \text { YR 5/4,5 } \\
10 \text { YR 5/5 }\end{array}$ & Argila arenosa & Lig. Peg. Lig. Plas. & F. P. M. & Argila Arenosa \\
\hline
\end{tabular}

BA ou perda por movimento lateral, o que deverá contribuir para acelerar o processo erosivo nesses ambientes quando trabalhado excessivamente.

Em todos os perfis analisados, de maneira geral, a densidade do solo variou de 1,19 a 1,67 kg. $\mathrm{dm}^{-3}$. Segundo Vieira et al. (2000), a densidade dos solos amazônicos geralmente encontra-se na faixa entre 1,2 a 1,8 kg. $\mathrm{dm}^{-3}$. Nota-se que o perfil 1-paricá com resíduo e o perfil 3-pupunha com resíduo apresentaram nos horizontes Ap menor densidade, na ordem de 1,19 kg. $\mathrm{dm}^{-3}$ a 1,33 $\mathrm{kg} \cdot \mathrm{dm}^{-3}$, respectivamente. A menor densidade do solo nos horizontes superficiais pode estar relacionado com a maior concentração de resíduo de madeira, originada da adição do material orgânico adicionado ao solo.
De acordo com Costa (1983), solos cultivados com adição de resíduos orgânicos mostram que a matéria orgânica sobre o efeito marcante na densidade do solo pode ser usada para predizer o valor desta característica física. Em solos arados e gradeados, 50\% da variação na densidade do solo seriam explicados pela variação do conteúdo de carbono nas camadas superficiais do solo.

Os valores da densidade de partículas dos solos não apresentam variação marcante ao longo do perfil nas profundidades estudadas, sendo o maior valor absoluto encontrado no horizonte BA no perfil P2-paricá sem resíduos. Estes valores encontrados para a densidade de partículas concordam, em parte, com os resultados encontrados por Jorge (1973), Costa e Teixeira (1992) e Vieira et al. (2000).

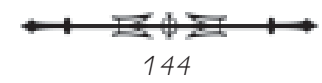


Segundo Buckman e Brady (1974), na maior parte dos solos minerais a densidade de partícula varia de 2,6 a 2,7 kg. $\mathrm{dm}^{-3}$, o que reflete a grande influência da presença dominante do quartzo, que apresenta peso específico de $2,65 \mathrm{~kg} \cdot \mathrm{dm}^{-3}$ (Tabela 2). A presença de óxidos de $\mathrm{Fe}$ e metais pesados aumentam o valor da Dp, enquanto que a matéria orgânica contribui para o seu decréscimo, o que justifica o menor valor encontrado nas camadas superficiais dos perfis com incorporação de resíduos de madeira. Assim, nos horizontes superficiais dos perfis 1-paricá com resíduos e 3-pupunha com resíduo, a Dp apresentou comportamento similar com valores $2,53 \mathrm{~kg} \cdot \mathrm{dm}^{-3}$ a $2,48 \mathrm{~kg} \cdot \mathrm{dm}^{-3}$ nos horizontes Ap e BA menores do que o perfil 2-paricá sem resíduos e perfil 4-pupunha sem resíduos.

A presença de resíduos influenciou a porosidade total nos horizontes superficiais (Ap, $\mathrm{BB}$ e $\mathrm{BA}$ ) para a área sob a cultura do paricá, enquanto que para a pupunha, essa influência parece estar limitada ao Ap. Nos perfis 2-paricá sem resíduo e 3-pupunha sem resíduo, houve redução no valor desta variável de $0,38 \mathrm{~m}^{3} \cdot \mathrm{m}^{-3}$ a $0,4 \mathrm{~m}^{3} \cdot \mathrm{m}^{-3}$, conforme já constatado para a densidade. Nos horizontes subjacentes, respectivamente, com valores de $0,32 \mathrm{~m}^{3} \cdot \mathrm{m}^{-3} \mathrm{e}$ $0,34 \mathrm{~m}^{3} \cdot \mathrm{m}^{-3} \mathrm{de}$ porosidade, evidencia-se, também, a ocorrência da compactação nos solos, principalmente com presença marcante nos perfis 1, 2 e 3. A contribuição direta da matéria orgânica na densidade e porosidade total é considerável no favorecimento da maior exploração de solos através do melhor desenvolvimento do sistema radicular das plantas, principalmente em solos tropicais, cujo relacionamento é, de certa maneira, reconhecido por vários autores, entre eles Costa (1983), Kiehl (1984), Fuller e Warrick (1986), Luz, Santos e Mermut (1992) e Lima (2001).

Tabela 2. Características físicas dos solos sob as culturas de pupunha e paricá.

\begin{tabular}{|c|c|c|c|c|c|c|c|c|c|c|}
\hline Horiz. & $\begin{array}{l}\text { Prof. } \\
\mathrm{cm}\end{array}$ & $\begin{array}{l}\text { Areia } \\
\text { areia grossa }\end{array}$ & $\begin{array}{l}\mathrm{kg}^{-1} \\
\text { areia fina }\end{array}$ & $\begin{array}{l}\text { Arg } \\
\text { argila total }\end{array}$ & $\begin{array}{l}\text { ila } g . \mathrm{kg}^{-1} \\
\mathrm{ADA}^{*}\end{array}$ & Silte & $\begin{array}{l}\text { silte/argila } \\
\mathrm{Kg} \cdot \mathrm{dm}^{-3}\end{array}$ & $\begin{array}{l}\text { Densidade } \\
\text { Solo }\end{array}$ & $\begin{array}{l}\text { Densidade } \\
\text { Partícula }\end{array}$ & $\begin{array}{c}\text { Porosidade } \\
\mathrm{m}^{3} \cdot \mathrm{m}^{3}\end{array}$ \\
\hline \multicolumn{11}{|c|}{ PERFIL 1 - PARICÁ: ARGISSOLO AMARELO DISTRÓFICO TÍPICO, TEXTURA MÉDIA/ARGILOSA } \\
\hline Ap & $0-13 / 15$ & 440 & 310 & 160 & 40 & 90 & 0,56 & 1.19 & 2.58 & 48 \\
\hline$A B$ & $13 / 15-25$ & 400 & 280 & 220 & 100 & 100 & 0,5 & 1.66 & 2.50 & 41 \\
\hline BA & $25-45$ & 350 & 280 & 320 & 200 & 50 & 0,15 & 1.44 & 2.46 & 39 \\
\hline \multicolumn{11}{|c|}{ PERFIL 2- PARICÁ: ARGISSOLO AMARELO DISTRÓFICO TÍPICO, TEXTURA ARENOSA/ARGILOSA } \\
\hline$A_{p}$ & $0-10$ & 470 & 360 & 120 & 20 & 50 & 0,41 & 1.51 & 2.62 & 35 \\
\hline$A B$ & $10-25$ & 430 & 340 & 150 & 40 & 80 & 0,53 & 1.67 & 2.62 & 30 \\
\hline BA & $25-45$ & 350 & 270 & 290 & 200 & 90 & 0,31 & 1.67 & 2.62 & 32 \\
\hline \multicolumn{11}{|c|}{ PERFIL 3-PUPUNHA: ARGISSOLO AMARELO EUTRÓFICO TÍPICO, TEXTURA MÉDIA/ARGILOSA } \\
\hline AP & $0-7$ & 390 & 360 & 180 & 60 & 70 & 0,38 & 1.33 & 2.55 & 46 \\
\hline$A B$ & $7-20$ & 330 & 280 & 300 & 220 & 90 & 0,3 & 1.53 & 2.64 & 41 \\
\hline BA & $20-31$ & 270 & 250 & 400 & 280 & 80 & 002 & 1.51 & 2.65 & 44 \\
\hline \multicolumn{11}{|c|}{ PERFIL 4-PUPUNHA: ARGISSOLO AMARELO DISTRÓFICO TÍPICO, TEXTURA MÉDIA/ARGILOSA } \\
\hline$A_{p}$ & $0-10$ & 390 & 330 & 180 & 60 & 90 & 0,5 & 1.60 & 2.67 & 38 \\
\hline$A B$ & $10-26$ & 370 & 280 & 260 & 160 & 90 & 0,34 & 1.54 & 2.63 & 39 \\
\hline $\mathrm{BA}$ & $26-48$ & 320 & 220 & 380 & 160 & 80 & 0,21 & 1.48 & 2.65 & 43 \\
\hline
\end{tabular}

*ADA-argila dissolvida em água

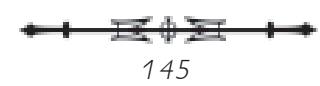




\section{Características Químicas}

Para os valores de matéria orgânica, o perfil 2 apresentou valores entre $5 \mathrm{~g} \cdot \mathrm{kg}^{-1} \mathrm{e} 9 \mathrm{~g} . \mathrm{kg}^{-1}$; enquanto que o perfil 4 variou de $5,6 \mathrm{~g} . \mathrm{kg}^{-}$a $12,22 \mathrm{~g} \cdot \mathrm{kg}^{-1}$, evidenciando-se, ainda nesta seqüência, tendência de diminuição com o aumento da profundidade para todos os perfis estudados (Tabela 3).

Já nos solos com resíduos de madeira, houve destaque para o perfil 3- pupunha, com valores de $6,7 \mathrm{~g} \cdot \mathrm{kg}^{-1}$ a $30 \mathrm{~g} \cdot \mathrm{kg}^{-1}$, enquanto que o perfil 1-paricá com resíduo atingiu valores de 5,4 g. $\mathrm{kg}^{-1}$ a 20,84 g. kg-1 no horizonte Ap, decrescendo acentuadamente nos horizontes subsuperficiais.

O maior conteúdo de matéria orgânica com diferentes coberturas sob diferentes sistemas de manejo foram encontrados também por Muzilli (1996), Woods, Meccani e Meyer (2000) e Falesi e Galeão (2002). Ainda de acordo com estes autores, estas práticas de manejo do solo com adição de material orgânico favoreceram ao incremento de matéria orgânica nos horizontes superficiais, principalmente se comparado com solos de florestas.
Os valores de cálcio + magnésio para os perfis sem adição de resíduos de madeira mostraram para o perfil 2 valores de 1,3 a 2,2 $\mathrm{cmol}_{c} \cdot \mathrm{dm}^{-3}$ e, para o perfil 4, valores de 1,6 a $2 \mathrm{cmol}_{c^{\prime}} \mathrm{dm}^{-3}$ nos horizontes superficiais, decrescendo em profundidade. A distribuição dos valores de cálcio + magnésio nos solos com adição de resíduos de madeira alcançaram para perfil 1-paricá com resíduo valores de 1,4 a 3,5 $\mathrm{cmol}_{c^{\prime}} \mathrm{dm}^{-3}$ e perfil 3-pupunha com resíduo resultados de 2,5 a 3,6 $\mathrm{cmol}$. $\mathrm{dm}^{-3}$. Os valores médios de cálcio + magnésio, encontrados nos perfis com adição de resíduo, demonstraram que a contribuição do material adicionado ao solo teve contribuição em elevar esta característica (Tabela 3).

Os valores encontrados para o pH nos solos com adição de resíduos de madeira alcançaram variação de 5,6 a 6 ao longo de todo o perfil 3, já o perfil 1 obteve variação de 3,7 a 4; enquanto que nos solos sem adição de resíduos, estes valores foram de 3,7 a 4,3 para o perfil 2, e variação de 4,8 a 5,2 para o perfil 4 .

Os resultados revelaram que os valores de $\mathrm{pH}$ para os perfis 1 e 3, ambos com adição de resíduos de madeira,

Tabela 3. Características químicas gerais dos solos da cultura de paricá e pupunha com e sem resíduo de madeira.

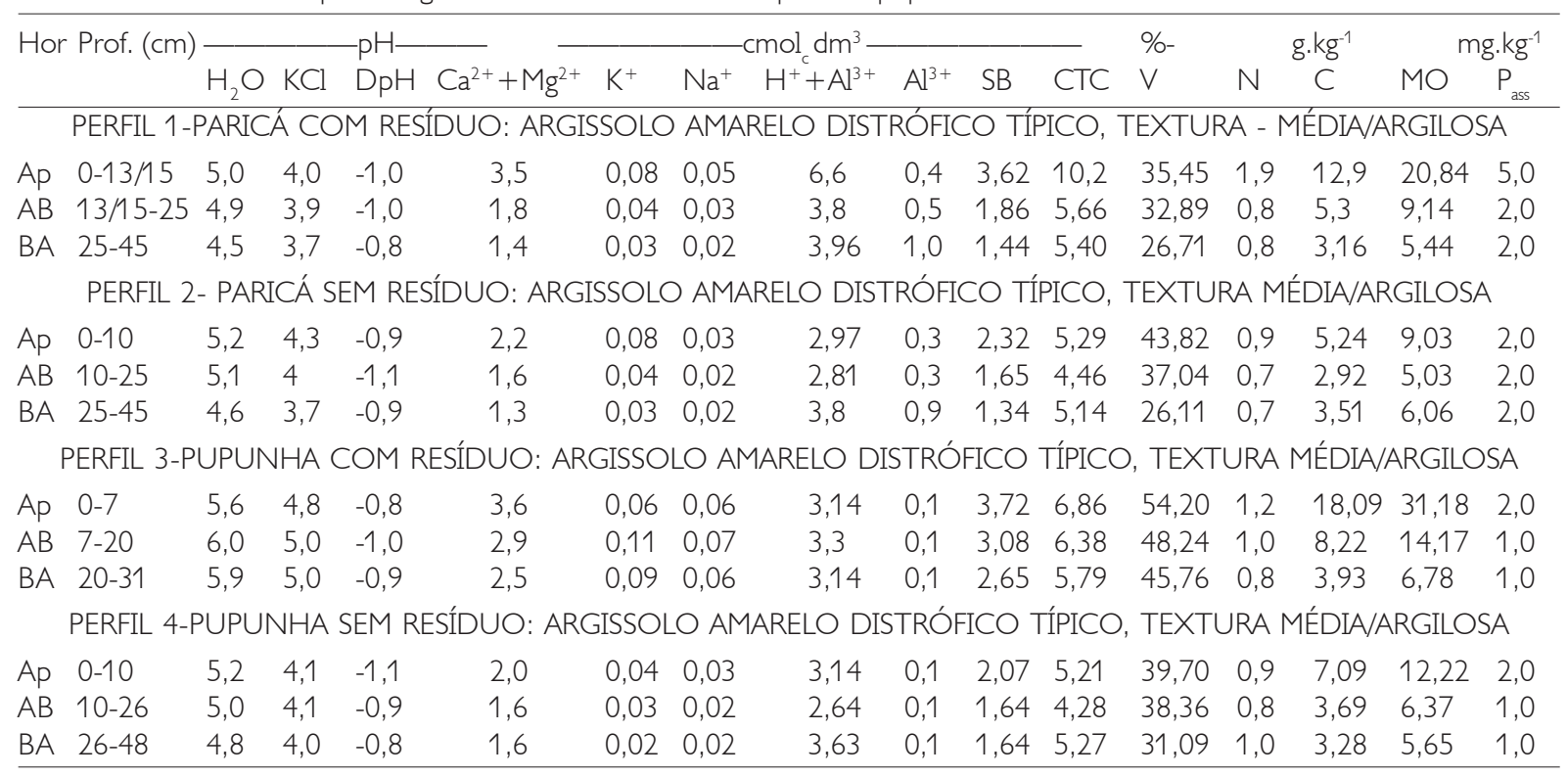

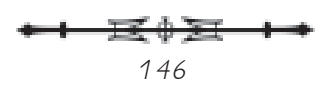


foram significativamente superiores. Estes resultados indicam a eficiência da matéria orgânica adicionada na área de pupunha, proporcionando diminuição considerável de sua acidez nas profundidades estudadas, podendo estar relacionada às concentrações de $\mathrm{Ca}^{2+}$ nestes solos a partir do material vegetal utilizado como cobertura. $\bigcirc$ que pode ser explicado, também, pela presença de cargas dependentes de $\mathrm{pH}$ e estas associadas diretamente à matéria orgânica, originadas da associação e dissociação do íon de $\mathrm{H}^{+}$ com radicais dos grupos carboxílicos e fenólicos (PRIMAVESI, 1988; RAIJ, 1991).

Os resultados encontrados para a soma de bases foram considerados baixos (Tabela 3). Verifica-se que, nas profundidades estudadas, o perfil 1 alcançou variação de 1,4 a 3,6 $\mathrm{cmol}_{c} \mathrm{~kg}^{-1}$, decrescendo em profundidade. Em relação ao perfil 3, este alcançou valores de 2,6 $\mathrm{cmol}_{\mathrm{c}} \cdot \mathrm{kg}^{-1}$ a $3,7 \mathrm{cmol}_{\mathrm{c}} \cdot \mathrm{kg}^{-1}$, decrescendo em profundidade e, no perfil 2 , os valores variaram de 1,3 a 2,3 $\mathrm{cmol}_{\mathrm{c}} \mathrm{kg}^{-1}$, com os menores valores nos horizontes subsuperficiais. Para o perfil 4, verificou-se valores de 1,6 a 2,7 $\mathrm{cmol}_{c^{\prime}} \mathrm{kg}^{-1}$.

Segundo Abreu Jr., Muraoka e Oliveira (2001), estes valores admitem que as classes destes solos apresentam razoáveis capacidades de troca de cátions no solo, representando, portanto, a graduação da liberação de vários nutrientes, o que favorece, de certa maneira, a manutenção da fertilidade por um prolongado período e reduz ou evita a ocorrência tóxica de outros elementos no solo.

Quanto ao índice de saturação por bases (V\%) em função das profundidades estudadas, notou-se que os valores alcançaram uma variação de 35,45 a 54,20\% para os perfis com adição de resíduos de madeira. Os solos sem incorporação de resíduos de madeira apresentaram valores de 39,7 a 43,72\%, perfil 2-paricá sem resíduo. Para o perfil 4, este também apresentou menor valor com no horizonte Ap a 31,74\% no horizonte subsuperficiais. Percebe-se que 0 comportamento dos solos sem adição de resíduos de madeira foi significativamente inferior aos solos onde não foram incorporados resíduos de madeira. O manejo do solo com utilização de resíduos de madeira nos argissolos estudados demonstrou ser uma prática benéfica e de uso racional, envolvendo o setor madeireiro e o setor agrícola na Amazônia.

\section{CONCLUSÃO}

O manejo dos Argissolos na região de Tailândia, Pará, utilizando resíduos de madeira como cobertura no solo, mostrou-se eficiente na melhoria das propriedades físicas como a densidade e a porosidade. Nas propriedades químicas, houve aumento nos valores de $\mathrm{pH}, \mathrm{V}, \mathrm{CTC}, \mathrm{SB}$, que são considerados indicadores de uma melhor condição de fertilidade química dos solos.

Através desta prática de manejo do solo ficou expresso que houve maior incremento de matéria orgânica através da utilização de resíduos de madeira como cobertura no solo, contribuindo para o aporte deste material nos solos da região amazônica, considerados pobres em material orgânico.

A experiência da empresa de laminação Tailâminas Plac demonstra uma nova e promissora abordagem do setor madeireiro referente às questões ambientais na Amazônia e vem somar esforços com o poder público na busca de uma melhor qualidade de vida para a população da região. Desta maneira, a atividade madeireira, ao deixar de incinerar, concede uma destinação adequada aos seus resíduos, utilizando-os como cobertura no solo.

\section{AGRADECIMENTOS}

Ao empresário Sr. Gilberto Sufredini, proprietário da empresa de laminação Tailâminas Plac Ltda; ao Programa de Formação Pessoal e Fortalecimento Institucional da Agência de Desenvolvimento da Amazônia (ADA); ao Fundo Estadual de Ciência e Tecnologia (FUNTEC) do estado do Pará, pelo apoio e cooperação para a realização deste trabalho.

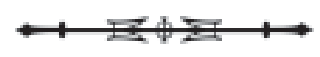




\section{REFERÊNCIAS}

ABREU JR., C. H.; MURAOKA, T.; OlVEIRA, F. C. 2001. Cátions trocáveis, capacidade de troca de cátions e saturação por bases em solos brasileiros adubados com compostos de lixo urbano. Scientia Agrícola, v. 58, n. 4, p. 813-824.

BELLO. A.; PÉREZ-LOPES. J.; ÀlVAREZ.G.A. 2003. Biofumigacion del Suelo, Residuos Orgánicos y Conservación de la Capa de Ozono. Disponivel: <www. Agroecologia.net/hacemos/ biofumigacion-suelo>. Acesso em: 20 ago.

BUCKMAN, H. O.; BRADY, N. C. 1983. Natureza e propriedades do solos. 6. ed. Rio de Janeiro: Freitas Bastos. $647 \mathrm{p}$.

COSTA, M. P. da. 1983. Efeito da matéria orgânica em alguns atributos do solo. 137 f. Dissertação (Mestrado) ESALQ-USP, Piracicaba, São Paulo.

COSTA, M. P. da; TEIXEIRA, L. B. 1992. Caracterização físicohídrica de Latossolo Amarelo da Região de Capitão PoçoPA. Belém: EMBRAPA-CPATU. 23 p. (Boletim de pesquisa EMBRAPACPATU, 133).

EMBRAPA. 1999. Empresa Brasileira de Pesquisa Agropecuária. Serviço Nacional de Levantamento e Conservação de Solos. Manual de análises químicas de solos, plantas e fertilizantes. Rio de Janeiro: [EMBRAPA]. $370 \mathrm{p}$.

FALESI, I. C.; GALEÃO, R. R. 2002. Recuperação de Áreas Antropizadas da Mesorregião Nordeste Paraense através de Sistemas Agroflorestais. Belém: EMATER-PA. 25 p. (Documentos, n. 1).

FULLER, W. H.; WARRICK, A. W. 1986. Soil in Wast Treatment and Utilizatin. Land Treatment, Pollutant Containment, Monitoring and Closure. Boca Raton, Flórida: CRC Press. 268 p. v. $1 / 2$

FELLER, C.; BEARE, M. H. 1997. Physical control of soil organic matter dynamicx in the tropics. Geoderma, v. 79, p. 69-116.

GREENLAND, D. J. 1981. Soil management and soil degradation. Journal of Soil Science, Reading, v. 32, p. 301-322.
JORGE, J. A. 1973. Física e Manejo dos Solos Tropicais. Campinas, São Paulo: Instituto Campineiro de Ensino Agrícola.

KIEHL, E. J. 1984. Efeitos da Matéria Orgânica sobre as Propriedades do Solo. In: SIMPÓSIO SOBRE FERTILIZANTES ORGÂNICOS, 1984, São Paulo. Anais... São Paulo: IPT, FINEP, ESALQ. p. 3-18. LEMOS, R. C.; SANTOS, R. D. 1996. Manual de descrição e coleta do solo no campo. Campinas: SBCS, SNLCS. 83 p.

LUZ, L. R. Q. P.; SANTOS, M. C.; MERMUT, A. R. 1992. Pedogênese em uma topossequência do semi-árido de Pernambuco. Revista Brasileira de Ciência do Solo, v. 16, p. 95-102.

MUZILLI, O. 1996. A fertilidade do solo no contexto da agricultura sustentável. In: CONGRESSO LATINO-AMERICANO DE CIÊNCIA DO SOLO, 12., 1996, Águas de Lindóia (SP).. Anais... São Paulo: Comissão de Fertilidade do Solo e Nutrição Mineral de Plantas.

PRIMAVESI, J. E. 1988. Fertilizantes Orgânicos. PiracicabaSão Paulo: Ed. Agronômica. 492 p.

RAIJ, B. V. 1991. Fertilidade do Solo e adubação. São Paulo: Ceres. Potafos. 343 p.

RODRIGUES, T. E. 2001. Caracterização e Classificação dos solos do Município de Tailândia, estado do Pará. Belém: EMBRAPA-CPATU. 32 p. Relatório Técnico.

SANCHEZ, P. A. 1976. Soil organic matter. In: PROPERTIES and management of soils in the tropics. New York: J. Willey. Chap. 246 p. v. 5.

TOMÉ JR., J. B. 1997. Manual para interpretação de análise de solo. Guaíba: Agropecuária. 247 p.

VIEIRA, L. S. et al. 2000. Levantamento e conservação do solo. 2. ed. Belém: FCAP, Serviço de documentação e informação. $320 \mathrm{p}$

WOODS, W. I.; MCCANN, J. M.; MEYER, D. W. E. 2000. Amazonian Dark Earth Analysis: state of knowledge and directions for future research. Paper and Procedings of the Applied Geography Conferences, v. 23, p. 110-120.

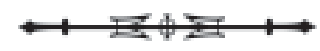

